\title{
Associations of left ventricular systolic dysfunction with the factors among Thai patients on peritoneal dialysis: a cross- sectional study
}

Teeranan Angkananard ${ }^{1}$, Jirayut Janma ${ }^{2}$, Thanapath Wannasiri ${ }^{1}$, Piyathida Sangthong ${ }^{1}$ and Siribha Changsirikulchai ${ }^{2^{*}}$ (D)

\begin{abstract}
Background: Factors associated with left ventricular systolic dysfunction (LVSD) of peritoneal dialysis (PD) patients are limited. We aim to explore and quantify the associated factors of LVSD among PD patients.

Methods: Participants from a PD clinic treated between 2012 and 2014 at the HRH Princess Maha Chakri Sirindhorn Medical Center, Srinakharinwirot University, Nakhon Nayok, Thailand were recruited and divided into 2 groups according to their left ventricular ejection fraction (LVEF) $(<50 \%$ vs. $\geq 50 \%)$ with LVEF $<50 \%$ considered as LVSD. Correlations among the clinical, laboratory and echocardiographic variables were analyzed. The factors associated with LVSD were explored with univariate and multivariate logistic regression analyses. Beta coefficient along with odds ratio and $95 \%$ confidence interval $(\mathrm{Cl})$ were calculated and the $P$ value $<0.05$ was considered significant.

Results: Among 103 subjects stratified as LVSD ( $n=18,17.5 \%)$. The mean (SD) age was 59.3 (12.7) years, and nearly halves were males. Preexisting CAD, diabetes (DM) and current smoking were 20 (19.4\%), 63 (61.2\%) and 23 (22.3\%) patients, respectively. The median time of dialysis vintage was $12(3,24)$ months. Factors associated with LVSD and corresponding ORs with $95 \% \mathrm{Cl}$ by multivariate analysis were prior coronary artery disease (CAD) $[5.08(1.16,22.19)]$, DM [6.36 (1.29, 31.49)], smoking [10.62 $(2.17,51.99)]$, neutrophil to lymphocyte ratio (NLR) > 3.6 [6.77 (1.41, 32.52)], and high serum phosphate $[9.39(2.16,40.92)]$ were significantly associated with LVSD.

Conclusions: Prior history of CAD, DM, smoking, high NLR and serum phosphate levels were found to be associated with LVSD for our PD patients. The evidence from prospective study is needed to confirm the predictive value of these variables.
\end{abstract}

Keywords: Peritoneal dialysis, Left ventricular systolic dysfunction, Neutrophil to lymphocyte ratio, Platelet to lymphocyte ratio, End stage renal disease

\section{Background}

Cardiovascular diseases (CVDs) are the leading causes of death in patients with end stage renal disease (ESRD) or on dialysis rather than progress to ESRD [1]. Atherosclerotic heart disease and congestive heart failure are the most common conditions

\footnotetext{
* Correspondence: siribha@swu.ac.th; siribha@g.swu.ac.th

2Division of Nephrology, Department of Medicine, Faculty of Medicine, HRH Princess Maha Chakri Sirindhorn Medical Center, Srinakharinwirot University, Nakhon Nayok, Thailand

Full list of author information is available at the end of the article
}

and highest among patients performing dialysis [2, 3]. All causes of mortality increase six fold for ESRD patients with left ventricular systolic dysfunction (LVSD) [4]. The general population with preserved left ventricular systolic function was found to be a cardioprotective factor for cardiovascular morbidity [5]. Whereas, LVSD is a strong, unfavorable prognostic factor for hemodialysis (HD) and renal transplant patients [6-8]. The combination of established risk factors and left ventricular ejection fraction (LVEF) significantly increases the $\mathrm{C}$ index to

(c) The Author(s). 2019 Open Access This article is distributed under the terms of the Creative Commons Attribution 4.0 International License (http://creativecommons.org/licenses/by/4.0/), which permits unrestricted use, distribution, and 
anticipate both cardiovascular and all causes of mortality in HD patients [7]. The possible mechanisms are multifactorial. These include; preexisting ischemic heart disease, anemia, hyperparathyroidism, uremic toxins, an increased serum calcium-phosphate product, malnutrition and prolonged hemodynamic volume overload [9]. LVSD was observed in all dialysis patients at $5-36 \%$ which varies by the mode of dialysis and the echocardiography study time. The associated factors of LVSD in peritoneal dialysis (PD) patients are also limited $[4,7,8,10-14]$.

The inflammatory response is a key mechanism in the pathogenesis of atherosclerosis and its related clinical syndromes. Prior studies have proposed that inflammation may be responsible for LVSD and left ventricular diastolic dysfunction in PD patients $[15,16]$. The neutrophil to lymphocyte ratio (NLR) and platelet to lymphocyte ratio (PLR), which are acquired from routine laboratory tests, have recently emerged as inflammatory biomarkers in ESRD patients [17, 18]. They have been found as useful predictors for LVSD in patients with acute coronary syndrome $[19,20]$. Currently, there is no data of NLR and PLR for predicting LVSD in PD patients. The objectives of this study are those we have conducted a cross-sectional analysis to explore and quantify associated clinical factors and laboratory data, including NLR and PLR in conjunction with echocardiographic parameters, in PD patients with LVSD.

\section{Methods}

\section{Study population}

All PD patients treated between 2012 and 2014 from the PD clinic at the HRH Princess Maha Chakri Sirindhorn Medical Center, Srinakharinwirot University, Nakhon Nayok, Thailand were recruited. Our PD patients were prescribed only glucose-based PD solution. The most percentage of glucose-based PD solution was $1.5 \%$ dextrose. The 2.5 and $4.25 \%$ dextrose-based PD solution could be in a short term if patients had severe hypervolemia. The criteria for eligibility were patients at least 18 years of age without history of heart failure or pulmonary embolism, valvular heart disease, congenital heart disease, acute coronary syndrome, chronic lung and liver diseases, systemic lupus erythematosus, scleroderma, initiating PD treatment $\leq 2$ weeks and infection during 3 months prior entry into this study. These data were verified by the physicians who were involved in our protocol. All patients have given their written, informed consent.

\section{Data collection}

Baseline demographic and clinical data were collected. These include; age, gender, body mass index (BMI), body surface area (BSA), underlying diseases [CAD, hypertension, diabetes (DM), hyperlipidemia, and pulmonary disease], duration of PD therapy (dialysis vintage in months), current medications, history of smoking and alcohol use, systolic blood pressure and diastolic blood pressure levels. CAD is defined as history of receiving angioplasty, coronary artery bypass graft surgery, myocardial infarction or angina. BMI was calculated as weight $/$ height $^{2}\left(\mathrm{~kg} / \mathrm{m}^{2}\right)$.

Trans-thoracic echocardiography [21] was performed on the same day of enrollment by a single cardiologist (first author) and one of two sonographers (the third and fourth authors) using the Philip iE33 xMATRIX echocardiographic system and the criteria set forth by the American Society of Echocardiography [22, 23]. Volumetric measurement was calculated using two apical views: four- and two-chamber views, and manual tracing of the endocardial border at the end-diastole and end-systole of both views. End-diastole could be determined as the frame following mitral valve closure or at the onset of the QRS complex while end-systole was defined as the frame prior mitral valve opening. LVEF was then computed as follows:

LVEF $=($ LV end diastolic volume-LV end systolic volume $\mid$ LV end diastolic volume $)$

By 2-D directed M-mode tracings, LV end-systolic diameter (LVESD) was estimated at end-systole, whereas LV posterior wall diastolic thickness, LV end-diastolic diameter (LVEDD) and interventricular septum diastolic thickness were determined at end-diastole. Left atrial (LA) volume and LA volume index (LAVI) were measured by biplane area-length method. Left ventricular mass was calculated by use of the Devereux formula [24]. Left ventricular hypertrophy, and pulmonary hypertension were diagnosed according to the current recommendation as stated in previous studies [23, 25]. LVEF was assessed by the biplane modified Simpson's method [26] and values less than 50\% were considered as LVSD according to a previous study [7]. LV diastolic function parameters were also collected, including mitral valve E/ A ratio, annular e' velocity, average mitral E/e' ratio, LAVI and peak tricuspid regurgitation (TR) velocity. The investigation of body composition, for example multifrequencies impedance meter, was not measured in this study. The laboratory parameters within 30 days of the TTE study were collected. These are; neutrophil, lymphocyte, platelet counts, hemoglobin $(\mathrm{Hb})$ levels, serum albumin, calcium, phosphate and estimated glomerular filtration rate (eGFR). The eGFR was analyzed using the simplified Modification of Diet in Renal Disease (MDRD) study equation [27] or the Chronic Kidney Disease Epidemiology Collaboration (CKD-EPI) equation [28]. We measured NLR by dividing absolute neutrophil count with absolute lymphocyte count while the PLR was calculated by 
dividing absolute platelet with lymphocyte count. two groups, $\leq 3.6$ vs $>3.6$ and $\leq 150$ vs $>150$, respectBased on previous studies [20,29], we used limits of ively. The corrected QT interval was also calculated 3.6 and 150 of NLR and PLR for categorizing into with 12-lead electrocardiography using Bazett's

Table 1 Baseline demographic, laboratory and echocardiographic characteristics

\begin{tabular}{|c|c|c|c|c|c|}
\hline $\begin{array}{l}\text { Demographic and clinical } \\
\text { characteristics }\end{array}$ & $\begin{array}{l}\text { Total } \\
n=103\end{array}$ & $\begin{array}{l}\mathrm{NLR}>3.6 \\
n=41\end{array}$ & $\begin{array}{l}N L R \leq 3.6 \\
n=62\end{array}$ & $\begin{array}{l}\text { PLR }>150 \\
n=31\end{array}$ & $\begin{array}{l}P L R \leq 150 \\
n=72\end{array}$ \\
\hline Age, y & $59.3 \pm 12.7$ & $59.61 \pm 13.06$ & $59.13 \pm 12.58$ & $57.65 \pm 12.52$ & $60.04 \pm 12.82$ \\
\hline Male, n (\%) & $50(48.5)$ & $21(51.22)$ & $29(46.77)$ & $20(64.52)$ & $30(41.67)$ \\
\hline Diabetes Mellitus, n (\%) & $63(61.2)$ & $28(68.29)$ & $35(56.45)$ & $23(74.19)$ & $40(55.56)$ \\
\hline Hypertension, n (\%) & $101(98.1)$ & $40(97.56)$ & 61 (98.39) & $30(96.77)$ & $71(98.61)$ \\
\hline Dyslipidemia, n (\%) & $78(75.7)$ & $30(73.17)$ & $48(77.42)$ & 19 (61.29) & $59(81.94)$ \\
\hline Smoker, n (\%) & $23(22.3)$ & $4(9.76)$ & $19(30.65)$ & $8(25.81)$ & $15(20.83)$ \\
\hline Previous CAD, n (\%) & $20(19.4)$ & $11(26.83)$ & $9(14.52)$ & $5(16.13)$ & $15(20.83)$ \\
\hline Pulmonary disease, n (\%) & $2(1.9)$ & 0 & $2(3.23)$ & $1(3.23)$ & $1(1.39)$ \\
\hline $\mathrm{SBP}, \mathrm{mmHg}$ & $150.7 \pm 23.0$ & $148.54 \pm 21.27$ & $152.19 \pm 24.15$ & $144.03 \pm 18.77$ & $153.63 \pm 24.16$ \\
\hline $\mathrm{DBP}, \mathrm{mmHg}$ & $82.6 \pm 12.7$ & $83.54 \pm 15.49$ & $82.03 \pm 10.62$ & $81.55 \pm 13.55$ & $83.09 \pm 12.43$ \\
\hline $\mathrm{BMI}, \mathrm{Kg} / \mathrm{m}^{2}$ & $24.2 \pm 3.6$ & $23.57 \pm 3.65$ & $24.59 \pm 3.62$ & $23.77 \pm 3.18$ & $24.36 \pm 3.84$ \\
\hline BSA, $\mathrm{m}^{2}$ & $1.20 \pm 0.10$ & $1.19 \pm 0.09$ & $1.18 \pm 0.09$ & $1.22 \pm 0.09$ & $1.18 \pm 0.09$ \\
\hline Duration of CAPD, months ${ }^{a}$ & $12.0(3.0,24.0)$ & $11.0(4.0,23.0)$ & $12.0(3.0,24.0)$ & $9.0(2.0,18.0)$ & $13.5(4.25,28.50)$ \\
\hline \multicolumn{6}{|l|}{ Blood biochemistry } \\
\hline$N L R^{a}$ & $3.3(2.3,4.3)$ & $4.9(3.9,5.8)$ & $2.5(2.0,3.2)$ & $5.2(3.4,8.8)$ & $2.9(2.2,3.6)$ \\
\hline$P L R^{a}$ & $164.1(119.0,231.6)$ & $168.1(109.8,246.9)$ & $97.1(68.9,128.5)$ & $203.5(168.75,271.8)$ & $95.9(68.8,120.7)$ \\
\hline $\mathrm{Hb} ; \mathrm{g} / \mathrm{dl}$ & $10.4 \pm 1.8$ & $10.4 \pm 1.8$ & $10.4 \pm 1.8$ & $10.2 \pm 2.1$ & $10.6 \pm 1.6$ \\
\hline Serum creatinine; $g / d L$ & $8.9 \pm 3.1$ & $8.7 \pm 2.8$ & $9.1 \pm 3.3$ & $8.9 \pm 2.9$ & $8.9 \pm 3.2$ \\
\hline GFR (CKD-EPI) $)^{\mathrm{a}} ; \mathrm{ml} / \mathrm{min} / 1.73 \mathrm{~m}^{2}$ & $5.3(4.0,6.9)$ & $6.1 \pm 2.9$ & $6.1 \pm 5.0$ & $6.1 \pm 2.7$ & $6.1 \pm 4.8$ \\
\hline Serum albumin; g/dL & $3.4 \pm 0.5$ & $3.2 \pm 0.5$ & $3.6 \pm 0.5$ & $3.1 \pm 0.6$ & $3.6 \pm 0.5$ \\
\hline Serum calcium; mg/dL & $8.4 \pm 0.9$ & $8.2 \pm 1.1$ & $8.6 \pm 0.8$ & $8.0 \pm 0.9$ & $8.6 \pm 0.9$ \\
\hline Serum phosphate; mg/dL & $4.8 \pm 1.5$ & $4.6 \pm 1.4$ & $4.9 \pm 1.5$ & $4.9 \pm 1.6$ & $4.7 \pm 1.4$ \\
\hline QTc interval; msec & $459.5 \pm 43.2$ & $460.4 \pm 48.6$ & $458.9 \pm 39.5$ & $468.6 \pm 47.8$ & $455.5 \pm 40.7$ \\
\hline \multicolumn{6}{|l|}{ Echocardiographic parameters } \\
\hline LVSD & $18(17.48)$ & $11(26.83)$ & $7(11.29)$ & $9(29.03)$ & $9(12.50)$ \\
\hline LVEF ${ }^{b} ; \%$ & $59.5+10.9$ & $56.0 \pm 12.3$ & $61.7 \pm 9.4$ & $55.2 \pm 11.2$ & $61.3 \pm 10.4$ \\
\hline LVEDD; mm & $49.5 \pm 8.3$ & $49.6 \pm 8.6$ & $49.5 \pm 8.2$ & $50.9 \pm 8,1$ & $48.9 \pm 8.4$ \\
\hline LVESD; mm & $32.9 \pm 8.5$ & $34.5 \pm 9.3$ & $31.9 \pm 7.8$ & $34.9 \pm 8.9$ & $32.1 \pm 8.2$ \\
\hline $\mathrm{LAVl} ; \mathrm{ml} / \mathrm{m}^{2}$ & $51.2 \pm 20.9$ & $53.7 \pm 28.7$ & $51.3 \pm 19.4$ & $53.4 \pm 22.1$ & $51.8 \pm 24.1$ \\
\hline LVMI; g/m² & $205.1 \pm 78.7$ & $206.9 \pm 79.2$ & $203.9 \pm 78.9$ & $216.8 \pm 82.3$ & $200.1 \pm 77.2$ \\
\hline IVSD; mm & $12.1 \pm 2.3$ & $12.2 \pm 2.3$ & $11.9 \pm 2.4$ & $12.4 \pm 2.7$ & $11.9 \pm 2.2$ \\
\hline IVSS; mm & $15.1 \pm 2.4$ & $15.1 \pm 2.5$ & $15.1 \pm 2.4$ & $15.5 \pm 2.9$ & $14.9 \pm 2.2$ \\
\hline Mitral E/A & $0.7(06,0.9)$ & $0.7(0.6,0.9)$ & $0.7(0.6,1.1)$ & $0.8(0.6,1.2)$ & $0.7(0.6,0.9)$ \\
\hline Mitral E/e' & $18.5 \pm 8.5$ & $20.4 \pm 10.9$ & $17.2 \pm 6.2$ & $19.4 \pm 10.4$ & $18.1 \pm 10.5$ \\
\hline RVSP; mmHg & $42.9 \pm 14.1$ & $43.6 \pm 15.6$ & $42.5 \pm 13.2$ & $45.4 \pm 14.7$ & $41.8 \pm 13.8$ \\
\hline TAPSE; cm & $2.3 \pm 0.5$ & $2.1 \pm 0.5$ & $2.4 \pm 0.5$ & $2.2 \pm 0.6$ & $2.4 \pm 0.5$ \\
\hline
\end{tabular}

Values are means $\pm S D,{ }^{a}$ values present as medians (IQR 25,75)

$C A D$ coronary artery disease, $d x$ disease, SBP systolic blood pressure, DBP diastolic blood pressure, BMI body mass index, BSA body surface area, CAPD continuous ambulatory peritoneal dialysis, NLR neutrophil lymphocyte ratio, PLR platelet lymphocyte ratio, Hb hemoglobin, GFR glomerular filtration rate, QTC Corrected QT interval= $Q T \sqrt{R R}$, LVSD left ventricular systolic dysfunction, $L V E F$ left ventricular ejection fraction, LVEF by modified Simpson's method, LVEDD left ventricular end diastolic diameter, LVESD left ventricular end systolic diameter, LAVI left atrial volume index, LVMI left ventricular mass index, IVSD interventricular septum in diastole, IVSS interventricular septum in systole, Mitral E/A mitral valve E velocity divided by A velocity, Mitral E/e' mitral valve E velocity divided by mitral annular e' velocity, RVSP right ventricular systolic pressure, TAPSE tricuspid annular plane systolic excursion 
Formula. Peritoneal membrane function was assessed using a standard peritoneal equilibration test [30] and the dialysis adequacy was shown as total weekly $\mathrm{Kt} / \mathrm{V}$ of urea.

\section{Statistical analysis}

Intra- and inter-observer reliability of TTE were analyzed prior to the initiation of this study. The continuous data was expressed as mean with standard deviation (SD) if normally distributed, or median with interquartile range if not. Subjects were divided into 2 groups of LVSD according to the cut off value of LVEF (<50\% vs. $\geq 50 \%)$. The categorical variables were presented as frequencies and percentages analyzed using Chi-square test or Fisher's exact test. Comparisons between groups of patients on continuous variables were performed using the Student's ttest or Mann-Whitney test. Pearson's and Spearman's rank correlation were applied to investigate the trend and strength of associations between LVSD and various risk factors. The univariate and multivariate logistic regression analyses were performed to determine significant factors associated with the categorical outcome variable of LVSD.

Data analysis was initiated with a univariate logistic regression analysis in order to screen for potential candidate variables and then added only these candidates; including NLR and PLR into the same model for multivariate analysis. Because of small sample size when compared with the number of variables needed, forward selection was used for considering which variables should or should not remain in the model. The backward elimination was not performed since it may force the model to over fit as for including all variables initially. Then the model selection was done by using "sw" command in STATA and keeping NLR and PLR as study factors in the model. Beta coefficient along with odds ratio and $95 \%$ confidence interval (CI) were calculated and the $P$ value $<0.05$ was considered significant. All statistical analysis was done using STATA 14.2 (StataCorp LP, College Station, TX, USA) software.

\section{Results}

Baseline clinical characteristics, laboratory and echocardiographic parameters are summarized in Table 1. Among 103 subjects, 50 (48.5\%) were male and the mean age was $59.3 \pm 12.7$ years. The participants were stratified into 2 groups according to their LVEF; i.e., group 1 with LVEF $\geq 50 \% \quad(n=85$, $82.5 \%)$ and group 2 with LVEF $<50 \%$ or LVSD $(n=$ $18,17.5 \%)$. The prevalence of LVSD was found 17.5\% (95\% CI, 11.21-26.21\%). Preexisting CAD, DM and current smoking were 20 (19.4\%), 63
(61.2\%) and $23(22.3 \%)$ patients, respectively. The mean BMI was $24.2 \pm 3.6 \mathrm{~kg} / \mathrm{m}^{2}$ and the median time of dialysis vintage was $12(3,24)$ months. Prior CAD $(p=0.02)$, DM $(p=0.03)$, smoking $(p=0.01)$ were significantly high while $\mathrm{Hb}$ levels were low in the PD patients with LVSD (Additional file 1: Table S1, Table S2). The median (IQR 25, 75) of PD adequacy was assessed by the total weekly $\mathrm{Kt} / \mathrm{V}$ of urea was $1.7(1.4,2.1)$. Dialysis efficiency data evaluated by total weekly $\mathrm{Kt} / \mathrm{V}$ of urea was not analyzed with other factors in the final because $50 \%$ of this data was missing.

The mean (SD) LVEF was 59.5\% (10.9) [63.2\% (7.4) versus $41.7 \%$ (6.80) in group 1 and group 2, respectively]. The left ventricular mass index (LVMI) $(p<0.01)$, LAVI $(p=0.03)$, and right ventricular systolic pressure (RVSP) $(p=0.01)$ were significantly higher in group 2 . The LVEF $\leq 40 \%$ was observed in only 7 of them $(6.8 \%)$ and that ranged from 21 to $40 \%$ (data not shown). LV dilatation was found in $12(14.1 \%)$ and $13(72.2 \%)$ patients of group 1 and 2, respectively. All patients of group 2 and $52(61.2 \%)$ patients of group 1 have met the criteria of elevated LV filling pressure and at least grade II of LV

Table 2 Correlation between LVEF and clinical and laboratory parameters

\begin{tabular}{lll}
\hline & $r$ & $P$-value \\
\hline Age, $y$ & +0.169 & 0.088 \\
SBP & +0.033 & 0.743 \\
DBP & -0.066 & 0.509 \\
BMI, Kg/m² & -0.024 & 0.813 \\
BSA, m ${ }^{2}$ & -0.027 & 0.784 \\
Duration of CAPD, months & +0.220 & 0.026 \\
NLR & -0.125 & 0.209 \\
PLR & -0.165 & 0.096 \\
Hb, g/dl & +0.236 & 0.017 \\
Serum creatinine, g/dL & -0.185 & 0.062 \\
GFR (MDRD); ml/min/1.73m ${ }^{2}$ & +0.147 & 0.139 \\
GFR (CKD-EPI); ml/min/1.73m ${ }^{2}$ & +0.128 & 0.196 \\
Serum albumin; g/dL & +0.188 & 0.058 \\
Serum calcium; mg/dL & +0.347 & 0.003 \\
Serum phosphate; mg/dL & -0.305 & 0.002 \\
QTc interval; msec & -0.128 & 0.197 \\
LVEDD; mm & -0.521 & $<0.001$ \\
LAVl; ml/m & -0.348 & $<0.001$ \\
LVMl; g/m ${ }^{2}$ & -0.456 & $<0.001$ \\
RVSP & -0.294 & 0.003 \\
\hline
\end{tabular}

LVEF left ventricular ejection fraction, SBP systolic blood pressure, DBP diastolic blood pressure, $B M I$ body mass index, BSA body surface area, CAPD continuous ambulatory peritoneal dialysis, NLR neutrophil lymphocyte ratio, $P L R$ platelet lymphocyte ratio, $H b$ hemoglobin, GFR glomerular filtration rate, $Q T C$ Corrected $Q T$ interval= $Q T \sqrt{R R}$, LVEDD left ventricular end diastolic diameter, $L A V I$ left atrial volume index, LVMI left ventricular mass index, RVSP right ventricular systolic pressure 
diastolic dysfunction [31]: average mitral E/e' ratio $>14$, LAVI $>34 \mathrm{ml} / \mathrm{m}^{2}$ and peak TR velocity $>2.8 \mathrm{~m} / \mathrm{sec}$.

Duration of dialysis $(\mathrm{r}=0.22, p=0.03)$, Hb level $(\mathrm{r}=$ $0.24, p=0.02)$ and serum calcium $(\mathrm{r}=0.35, p<0.01)$ showed a considerably positive correlation with LVEF. The serum phosphate $(\mathrm{r}=-0.31, \mathrm{p}<0.01)$, LVEDD $(\mathrm{r}=-0.52, \mathrm{p}<0.01)$, LAVI $(\mathrm{r}=-0.35, \mathrm{p}<0.01)$, LVMI $(\mathrm{r}=-0.46, \mathrm{p}<0.01)$ and RVSP $(\mathrm{r}=-0.29, \mathrm{p}<0.01)$ were significantly negative correlations (Table 2, Figs. 1 and 2).

The relationship between LVSD and associated factors were determined by univariate and multivariate logistic regression analysis. The LVSD was found to be associated with prior CAD, DM, smoking, Hb level, NLR > 3.6, PLR $>150$, high calcium and phosphate level (Table 3, all $P<0.05)$. However, in multivariate analysis showed only that the underlying CAD (OR 5.08, 95\% CI: 1.16, 22.19), DM (OR 6.36, 95\% CI: 1.29, 31.49), smoking (OR 10.62, 95\% CI: 2.17, 51.99), NLR > 3.6 (OR 6.77, 95\% CI: 1.41, 32.52), and high serum phosphate (OR 9.39, 95\% CI: $2.16,40.92)$ were significantly associated with LVSD (Table 3).
In addition, further analysis of PD patients with LVSD, prior CAD (OR 10.32, 95\% CI: 1.71, 62.14, $p=$ 0.01 ), and PLR $>150$ (OR 10.11, 95\% CI: 1.54, 66.26, $p=0.02$ ), were significant variable factors associated with LVEF $\leq 40 \%$ (Table 4).

\section{Discussion}

This study provides evidence that there is association between prior CAD, diabetes, smoking, high NLR and serum phosphate levels in PD patients with LVSD. There is correlation between inflammatory markers; i.e., NLR and PLR with LVSD of PD patients. The prevalence of LVSD among PD subjects was comparable with data seen in previous studies $[4,9,11,14]$. Prior CAD was a strong indicator for PD patients with LVEF $\leq 0.4$ which was similar to an earlier study [14]. However, the independent factors for LVSD in this study were different which could be explained by using a distinctive definition of LVSD (0.5 vs. 0.4) and enrolled participants were not only peritoneal dialysis (25\%), but also hemodialysis (75\%) patients [14]. We applied LVEF $<0.5$ as a cutoff value of LVSD in correspondence to a previous study
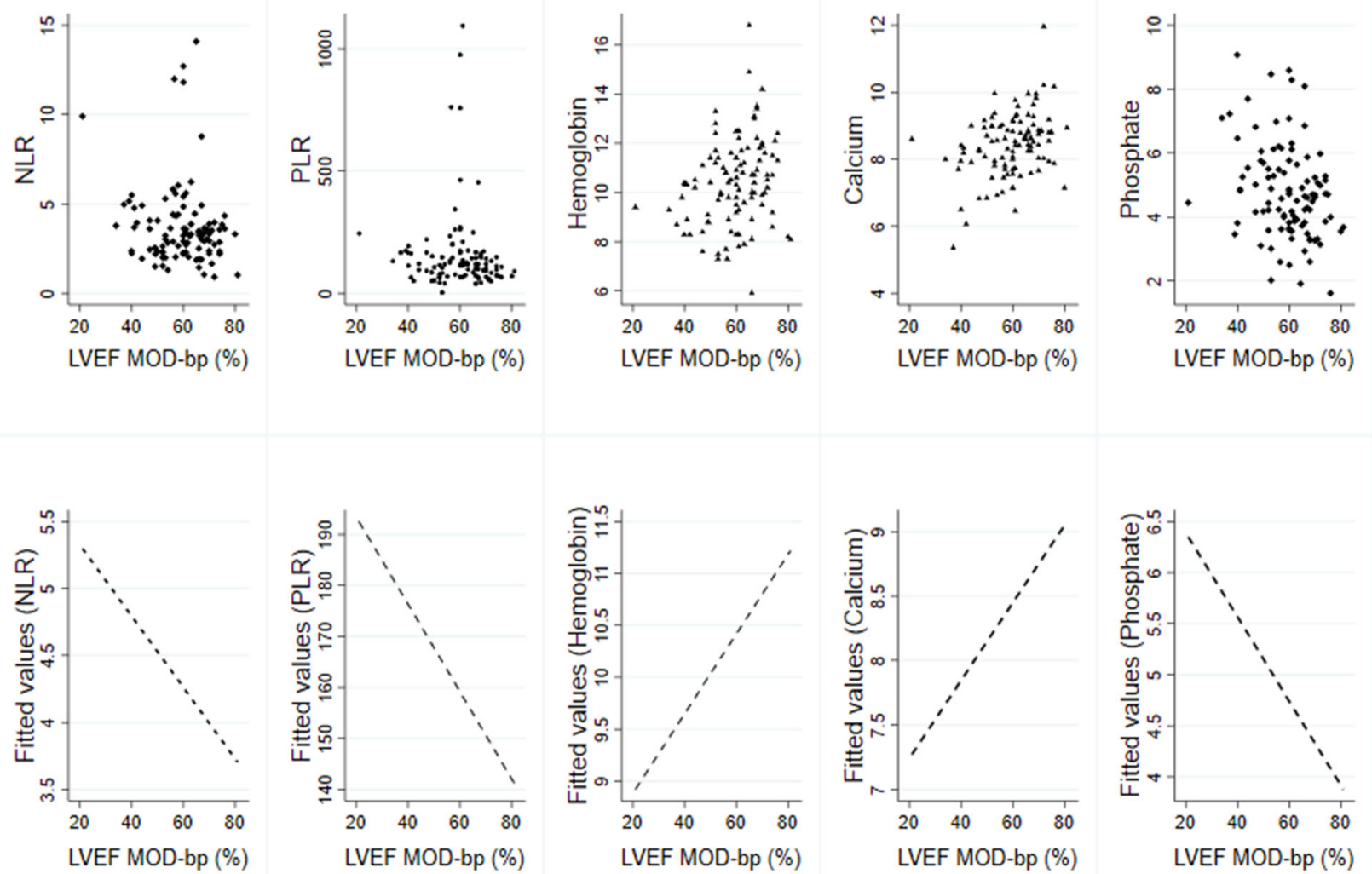

Fig. 1 Correlation between laboratory factors and left ventricular ejection fraction (LVEF), modified Simpson's method 



Fig. 2 Correlation between echocardiographic parameters and left ventricular ejection fraction (LVEF), modified Simpson's method

[7]. This value could significantly anticipate poor survival outcomes of ESRD patients for both cardiovascular and all causes of mortality. The progressive declining of LV systolic function could be prevented from early detection of LVSD.

Neutrophils, lymphocytes and monocytes play an important role in the systemic inflammatory response to severe injury and infection. A previous study investigated an immune response to endotoxemia and has demonstrated an increased amount of circulating neutrophils and a decreased number of lymphocytes [32]. It has been documented that inflammatory cytokines lead to heart failure progression and LV remodeling by generating hypertrophy and promoting myocyte apoptosis and fibrosis [16, 33]. These can explain the predictive role of NLR for the risk of LVSD.

The serum levels of calcium and phosphate were reported to be associated with LV diastolic function in ESRD patients $[34,35]$. These levels with LVSD has a similar correlation in our study. Hyperphosphatemia, observed in CKD patients, is associated with cardiac hypertrophy which may worsen cardiac contractility and heart failure [36]. Prior studies also demonstrate a relationship between serum calcium and phosphate levels with coronary, myocardial and valvular calcifications in uremic patients [37, 38]. Elevated serum phosphate levels may worsen the effects of coronary atherosclerosis through increased

Table 3 Factors associated with LVSD (LVEF < 50\%): Univariate and Multivariate analysis among PD patients

\begin{tabular}{|c|c|c|c|c|c|c|}
\hline \multirow[b]{2}{*}{ Factors } & \multicolumn{3}{|c|}{ Univariate analysis } & \multicolumn{3}{|c|}{ Multivariate analysis } \\
\hline & Odds Ratio & $95 \% \mathrm{Cl}$ & P-value & Odds Ratio & $95 \% \mathrm{Cl}$ & $P$-value \\
\hline Previous CAD & 3.524 & $1.154,10.766$ & 0.027 & 5.078 & $1.162,22.199$ & 0.031 \\
\hline Diabetes & 3.854 & $1.038,14.308$ & 0.044 & 6.362 & $1.285,31.493$ & 0.023 \\
\hline Smoke & 3.733 & $1.263,11.039$ & 0.017 & 10.617 & $2.168,51.988$ & 0.004 \\
\hline$N L R>3.6$ & 2.881 & $1.011,8.207$ & 0.048 & 6.774 & $1.411,32.519$ & 0.017 \\
\hline$P L R>150$ & 2.864 & $1.008,8.132$ & 0.048 & - & - & - \\
\hline $\mathrm{Hb} \leq 10 \mathrm{~g} / \mathrm{dL}$ & 3.313 & $1.132,9.693$ & 0.029 & - & - & - \\
\hline Calcium $^{\mathrm{a}} ; \mathrm{mg} / \mathrm{dL}$ & 0.209 & $0.064,0.691$ & 0.010 & - & - & - \\
\hline Phosphate $^{\mathrm{b}} ; \mathrm{mg} / \mathrm{dL}$ & 4.306 & $1.404,13.209$ & 0.011 & 9.397 & $2.158,40.920$ & 0.003 \\
\hline
\end{tabular}

${ }^{a}$ serum calcium $\geq 8.5 \mathrm{mg} / \mathrm{dL}$, ${ }^{\mathrm{b}}$ serum phosphate $\geq 4.8 \mathrm{mg} / \mathrm{dL}$

LVSD left ventricular systolic dysfunction, $L V E F$ left ventricular ejection fraction, $P D$ peritoneal dialysis, DM diabetes, CAD coronary artery disease, NLR neutrophil lymphocyte ratio, PLR platelet lymphocyte ratio, $\mathrm{Hb}$ hemoglobin 
Table 4 Factors associated with LVSD (LVEF < 40\%): Univariate and Multivariate analysis among PD patients

\begin{tabular}{|c|c|c|c|c|c|c|}
\hline \multirow[b]{2}{*}{ Factors } & \multicolumn{3}{|c|}{ Univariate Analysis } & \multicolumn{3}{|c|}{ Multivariate Analysis } \\
\hline & Odds Ratio & $95 \% \mathrm{Cl}$ & P-value & Odds Ratio & $95 \% \mathrm{Cl}$ & $P$-value \\
\hline Previous CAD & 6.667 & $1.359,32.701$ & 0.019 & 10.316 & $1.712,62.142$ & 0.011 \\
\hline Diabetes & 1.638 & $0.302,8.877$ & 0.567 & - & - & - \\
\hline Smoke & 5.404 & $1.114,26.205$ & 0.036 & - & - & - \\
\hline$N L R>3.6$ & 4.167 & $0.768,22.606$ & 0.098 & - & - & - \\
\hline PLR $>150$ & 6.731 & $1.229,36.862$ & 0.028 & 10.107 & $1.542,66.261$ & 0.016 \\
\hline $\mathrm{Hb} \leq 10 \mathrm{~g} / \mathrm{dL}$ & 3.654 & $0.674,19.795$ & 0.133 & - & - & - \\
\hline Calcium $; \mathrm{ag} / \mathrm{dL}$ & 0.141 & $0.163,1.216$ & 0.075 & - & - & - \\
\hline Phosphate $^{\mathrm{b}} ; \mathrm{mg} / \mathrm{dL}$ & 1.788 & $0.379,8.432$ & 0.462 & - & - & - \\
\hline
\end{tabular}

${ }^{a}$ serum calcium $\geq 8.5 \mathrm{mg} / \mathrm{dL}$, ${ }^{\mathrm{b}}$ serum phosphate $\geq 4.8 \mathrm{mg} / \mathrm{dL}$

LVSD left ventricular systolic dysfunction, $L V E F$ left ventricular ejection fraction, PD peritoneal dialysis, DM diabetes, CAD coronary artery disease, NLR neutrophil lymphocyte ratio, PLR platelet lymphocyte ratio

vascular calcification and smooth muscle proliferation [39]. This calcification may change microcirculatory hemodynamics through increased extravascular resistance, compromised myocardial perfusion and induced LV diastolic and systolic dysfunction [34].

Anemia is frequently observed in patients with CKD and could be found more than $80 \%$ of patients with a creatinine clearance $<25 \mathrm{ml} / \mathrm{min} / 1.73 \mathrm{~m}^{2}$ [40]. Anemia is associated complexly to the relationship between CKD and CAD in "Cardio-Renal-Anemia syndrome" [41, 42]. We postulate that there is an association as anemia causes reduced oxygen carrying capacity and contributes to myocardial ischemia and abnormal adaptive cardiac remodeling. There is significant heterogeneity in $\mathrm{Hb}$ level at each stage of kidney disease. Therefore, it is possible that anemia represents not only declined eGFR but also results in inflammation [43, 44]. Chronic anemia cause volume overload, which results in ventricular dilatation. Consequently, the length of the sarcomeres increases for a better overlap between myofilaments. The thickness of the left ventricle increases to counter balance the increased radius and eccentric hypertrophy which can predict poor outcomes of CAD and life expectancy [45].

The LVEF measurement was partly influenced by volume overload. Patients with LVEF $<0.5$ had larger LVEDD, LVESD, LAVI and peak TR velocity, however in this study $61.2 \%$ of participants with LVEF $\geq 0.5$ also had evidence of increased LV filling pressure, but nonsignificantly difference of LV dilatation.

This is the first study to address the association between these factors and LVSD in PD patients. Comparing with previous studies [10, 14], we investigated CKD patients on PD and not hemodialysis. To avoid confounding bias of LVSD outcomes, the echocardiographic factors are not included as the study factors in the final analysis. Our analysis indicated that NLR, PLR, Hb, serum calcium and phosphate levels, which are easily obtained from routine laboratory testing, may be useful for predicting and modifying the risk of LVSD in PD patients.

This study has some limitations; first, it is a crosssectional study which could not establish the causal relationship of LVSD among those variable factors. Secondly this study was done based on a single center and limited to PD patients, thus it has a small sample size for a small event of LVSD. This may be the reason of abnormally wide CI of OR. For accepting the final model and its validity, the goodness of fit was assessed and illustrated well fit with the given data. To reduce the width of a CI, the additional time, resources and budgets are needed to collect a larger sample. Lastly, C-reactive protein (CRP), high sensitivity CRP (hs-CRP) and other inflammatory markers are not included in our analysis as a result of the measurement of CRP and hs-CRP not widely used and rather expensive. NLR and PLR could be derived from a routine simple test; i.e., complete blood count. However, previous studies have illustrated the NLR could be a potential surrogate marker of systemic inflammation in its ability to predict hs-CRP [46] and CRP [47] levels of ESRD patients. In addition, the prognostic value of CRP as indicator of LVSD from previous studies $[48,49]$ have shown solely in hemodialysis patients. Future studies of CRP or hs-CRP levels in PD patients should be investigated as a prognostic marker for predicting LVSD of PD patients. In addition, further longitudinal studies which include more participants and centers are needed to explore this observed relationship for both modes of dialysis, peritoneal and hemodialysis, and for long term survival.

\section{Conclusion}

Prior history of CAD, DM, smoking, high NLR and serum phosphate levels were found to be associated with LVSD for our PD patients. The evidence from prospective study is needed to confirm the predictive value of these variables. 


\section{Additional file}

Additional file 1: Table S1. Baseline characteristics and Laboratory parameters in group 1 (normal LVEF) and group 2 (LVSD). Table S2. Echocardiographic parameters in group 1 (normal LVEF) and group 2 (LVSD). (DOCX $21 \mathrm{~kb}$ )

\section{Abbreviations}

BMI: Body mass index; BSA: Body surface area; CAD: Coronary artery disease; Cl: Confidence interval; CKD: Chronic kidney disease; CVD: Cardiovascular diseases; DM: Diabetes Mellitus; eGFR: estimated glomerular filtration rate; ESRD: End stage renal disease; Hb: Hemoglobin; HD: Hemodialysis; LA: Left atrium; LAVI: Left atrial volume index; LVEDD: Left ventricular end-diastolic diameter; LVEF: Left ventricular ejection fraction; LVESD: Left ventricular end systolic diameter; LVMI: Left ventricular mass index; LVSD: Left ventricular systolic dysfunction; MDRD: Modification of Diet in Renal Disease; NLR: Neutrophil to lymphocyte ratio; PD: Peritoneal dialysis; PLR: Platelet to lymphocyte ratio; RVSP: Right ventricular systolic pressure; SD: Standard deviation; TR: Tricuspid regurgitation; TTE: Trans-thoracic echocardiography

\section{Acknowledgements}

The authors are grateful to Assoc. Prof. Dr. Sasivimol Rattanasiri who is lecturer of Section for Clinical Epidemiology and Biostatistics, Faculty of Medicine, Ramathibodhi Hospital, Mahidol University, Thailand for statistical analysis and all staffs of the cardiovascular and CAPD units, Faculty of Medicine, the Her Royal Highness Princess Maha Chakri Sirindhorn Medical Center (MSMC), Srinakharinwirot University, Nakhon Nayok, Thailand. Mr. Robert Cho has also been acknowledged for manuscript preparation.

\section{Authors' contributions}

TA - conceived of the study, participated in its design, coordination, carried out echocardiography, analyzed and interpreted data and was a major contributor in writing the manuscript; JJ -participated in its design, coordination and wrote the manuscript; TW - carried out echocardiography; PS carried out echocardiography, participated in its design and coordination; SC- conceived of the study, participated in its design, coordination, wrote the manuscript, and revised the manuscript. All authors read and approved the final manuscript

\section{Funding}

Faculty of Medicine, HRH Princess Maha Chakri Sirindhorn Medical Center, Srinakharinwirot University, Nakhon Nayok, Thailand.

\section{Availability of data and materials}

All data used and/or analysed during the current study are presented in the manuscript or available from the corresponding author on reasonable request.

\section{Ethics approval and consent to participate}

The study was approved by the Institutional Review Board of Ethical Committee, Srinakharinwirot University, Thailand and implemented in accordance with the ethical standards of the 1964 Declaration of Helsinki and its later amendments. All participants in the study were voluntary and gave their written informed consent.

\section{Consent for publication}

Not applicable.

\section{Competing interests}

The authors declare that they have no competing interests.

\section{Author details}

${ }^{1}$ Division of Cardiovascular Medicine, Department of Medicine, Faculty of Medicine, HRH Princess Maha Chakri Sirindhorn Medical Center, Srinakharinwirot University, Nakhon Nayok, Thailand. ²Division of Nephrology, Department of Medicine, Faculty of Medicine, HRH Princess Maha Chakri Sirindhorn Medical Center, Srinakharinwirot University, Nakhon Nayok, Thailand.
Received: 18 December 2018 Accepted: 9 June 2019

Published online: 12 July 2019

\section{References}

1. Thompson S, James M, Wiebe N, Hemmelgarn B, Manns B, Klarenbach S, et al. Cause of death in patients with reduced kidney function. J Am Soc Nephrol. 2015:26(10):2504-11.

2. Bhat TM, Afari ME, Garcia LA. Neutrophil lymphocyte ratio in peripheral vascular disease: a review. Expert Rev Cardiovasc Ther. 2016;14(7):871-5.

3. Kim H, Kim KH, Ahn SV, Kang S-W, Yoo T-H, Ahn HS, et al. Risk of major cardiovascular events among incident dialysis patients: a Korean national population-based study. Int J Cardiol. 2015;198:95-101.

4. de Mattos AM, Siedlecki A, Gaston RS, Perry GJ, Julian BA, Kew CE, et al. Systolic dysfunction portends increased mortality among those waiting for renal transplant. J Am Soc Nephrol : J Am Soc Nephrol. 2008;19(6):1191-6.

5. Refaat H, Sany D, Mohab A, Ezzat H. Comparing Dialysis modality and cardiovascular mortality in patients on hemodialysis and peritoneal Dialysis. Adv Perit Dial. 2016;32:22-31.

6. McGregor E, Jardine AG, Murray LS, Dargie HJ, Rodger RS, Junor BJ, et al. Preoperative echocardiographic abnormalities and adverse outcome following renal transplantation. Nephrol Dial Transplant. 1998;13(6):1499-505.

7. Yamada S, Ishii H, Takahashi H, Aoyama T, Morita Y, Kasuga H, et al. Prognostic value of reduced left ventricular ejection fraction at start of hemodialysis therapy on cardiovascular and all-cause mortality in end-stage renal disease patients. Clin J Am Soc Nephrol. 2010;5(10):1793-8.

8. Foley RN, Parfrey PS, Harnett JD, Kent GM, Murray DC, Barre PE. The prognostic importance of left ventricular geometry in uremic cardiomyopathy. J Am Soc Nephrol. 1995;5(12):2024-31.

9. Dyadyk OI, Bagriy AE, Yarovaya NF. Disorders of left ventricular structure and function in chronic uremia: how often, why and what to do with it? Eur J Heart Fail. 1999;1(4):327-36.

10. Greaves SC, Gamble GD, Collins JF, Whalley GA, Sharpe DN. Determinants of left ventricular hypertrophy and systolic dysfunction in chronic renal failure. Am J Kidney Dis. 1994;24(5):768-76.

11. Foley RN, Parfrey PS, Harnett JD, Kent GM, Martin CJ, Murray DC, et al. Clinical and echocardiographic disease in patients starting end-stage renal disease therapy. Kidney Int. 1995;47(1):186-92.

12. Harnett JD, Foley RN, Kent GM, Barre PE, Murray D, Parfrey PS. Congestive heart failure in dialysis patients: prevalence, incidence, prognosis and risk factors. Kidney Int. 1995;47(3):884-90.

13. Joki N, Hase H, Saijyo T, Tanaka Y, Takahashi Y, Ishikawa $H$, et al. Combined assessment of cardiac systolic dysfunction and coronary atherosclerosis used to predict future cardiac deaths after starting hemodialysis. Am J Nephrol. 2003;23(6):458-65.

14. Chinprateep B, Ratanasit N, Chanchairujira T. Prevalence and factors associated with left ventricular systolic dysfunction in end-stage renal disease patients on dialysis. J Med Assoc Thail. 2016;99(6):653-8.

15. Lee JK, Lin HH, Tsai CT, Chen JJ, Kuo CC, Lien YC, et al. Differential association of proinflammatory cytokines with left ventricular diastolic dysfunction in subjects with and without continuous ambulatory peritoneal dialysis. Nutr Metab Cardiovasc Dis. 2012;22(11):974-80.

16. Wu CK, Huang YT, Lin HH, Yang CY, Lien YC, Lee JK, et al. Dissecting the mechanisms of left ventricular diastolic dysfunction and inflammation in peritoneal dialysis patients. PLoS One. 2013:8(5):e62722.

17. Venkatraghavan L, Tan TP, Mehta J, Arekapudi A, Govindarajulu A, Siu E. Neutrophil lymphocyte ratio as a predictor of systemic inflammation - a cross-sectional study in a pre-admission setting. F1000Res. 2015;4:123.

18. Ahbap E, Sakaci T, Kara E, Sahutoglu T, Koc Y, Basturk T, et al. Neutrophil-tolymphocyte ratio and platelet-tolymphocyte ratio in evaluation of inflammation in end-stage renal disease. Clin Nephrol. 2016:85(4):199-208.

19. Bekler A, Gazi E, Yilmaz M, Temiz A, Altun B, Barutcu A, et al. Could elevated platelet-lymphocyte ratio predict left ventricular systolic dysfunction in patients with non-ST elevated acute coronary syndrome? Anatol J Cardiol. 2015;15(5):385-90.

20. Bekler A, Erbag G, Sen H, Gazi E, Ozcan S. Predictive value of elevated neutrophil-lymphocyte ratio for left ventricular systolic dysfunction in patients with non ST-elevated acute coronary syndrome. Pak J Med Sci. 2015;31(1):159-63

21. Malhotra R, Marcelli D, von Gersdorff G, Grassmann A, Schaller M, Bayh I, et al. Relationship of neutrophil-to-lymphocyte ratio and serum albumin levels 
with C-reactive protein in hemodialysis patients: results from 2 international cohort studies. Nephron. 2015;130(4):263-70.

22. Gottdiener JS, Bednarz J, Devereux R, Gardin J, Klein A, Manning WJ, et al. American Society of Echocardiography recommendations for use of echocardiography in clinical trials. J Am Soc Echocardiogr. 2004;17(10):1086-119.

23. Lang RM, Badano LP, Mor-Avi V, Afilalo J, Armstrong A, Ernande L, et al. Recommendations for cardiac chamber quantification by echocardiography in adults: an update from the American Society of Echocardiography and the European Association of Cardiovascular Imaging. J Am Soc Echocardiogr. 2015:28(1):1-39.e14.

24. Devereux RB, Alonso DR, Lutas EM, Gottlieb GJ, Campo E, Sachs I, et al. Echocardiographic assessment of left ventricular hypertrophy: comparison to necropsy findings. Am J Cardiol. 1986;57(6):450-8.

25. Galiè N, Humbert M, Vachiery J-L, Gibbs S, Lang I, Torbicki A, et al. 2015 ESC/ERS guidelines for the diagnosis and treatment of pulmonary hypertensionThe joint task force for the diagnosis and treatment of pulmonary hypertension of the European Society of Cardiology (ESC) and the European Respiratory Society (ERS): endorsed by: Association for European Paediatric and Congenital Cardiology (AEPC), International Society for Heart and Lung Transplantation (ISHLT). Eur Heart J. 2016;37(1):67-119.

26. Schiller NB, Acquatella H, Ports TA, Drew D, Goerke J, Ringertz H, et al. Left ventricular volume from paired biplane two-dimensional echocardiography. Circulation. 1979;60(3):547.

27. Levey AS, Bosch JP, Lewis JB, Greene T, Rogers N, Roth D. A more accurate method to estimate glomerular filtration rate from serum creatinine: a new prediction equation. Modification of diet in renal disease study group. Ann Intern Med. 1999;130(6):461-70.

28. Matsushita K, Mahmoodi BK, Woodward M, Emberson JR, Jafar TH, Jee SH, et al. Comparison of risk prediction using the CKD-EPI equation and the MDRD study equation for estimated glomerular filtration rate. JAMA. 2012 307(18):1941-51.

29. Park TJ, Cho YH, Chung HS, Hwang EC, Jung SH, Hwang JE, et al. Prognostic significance of platelet-lymphocyte ratio in patients receiving first-line tyrosine kinase inhibitors for metastatic renal cell cancer. SpringerPlus. 2016;5(1):1889.

30. Mehrotra R, Ravel V, Streja E, Kuttykrishnan S, Adams SV, Katz R, et al. Peritoneal equilibration test and patient outcomes. Clin J Am Soc Nephrol. 2015;10(11):1990-2001

31. Nagueh SF, Smiseth OA, Appleton CP, Byrd BF 3rd, Dokainish H, Edvardsen $T$, et al. Recommendations for the evaluation of left ventricular diastolic function by echocardiography: an update from the American Society of Echocardiography and the European Association of Cardiovascular Imaging. J Am Soc Echocardiogr. 2016;29(4):277-314.

32. Jilma B, Blann A, Pernerstorfer T, Stohlawetz P, Eichler HG, Vondrovec B, et al. Regulation of adhesion molecules during human endotoxemia. No acute effects of aspirin. Am J Respir Crit Care Med. 1999;159(3):857-63.

33. Finkel MS, Oddis CV, Jacob TD, Watkins SC, Hattler BG, Simmons RL. Negative inotropic effects of cytokines on the heart mediated by nitric oxide. Science. 1992;257(5068):387-9.

34. Ye M, Tian N, Liu Y, Li W, Lin H, Fan R, et al. High serum phosphorus level is associated with left ventricular diastolic dysfunction in peritoneal Dialysis patients. PLoS One. 2016;11(9):e0163659.

35. Galetta F, Cupisti A, Franzoni F, Femia FR, Rossi M, Barsotti G, et al. Left ventricular function and calcium phosphate plasma levels in uraemic patients. J Inter Med. 2005;258(4):378-84.

36. Neves KR, Graciolli FG, Dos Reis LM, Pasqualucci CA, MoysÉS RMA, Jorgetti V. Adverse effects of hyperphosphatemia on myocardial hypertrophy, renal function, and bone in rats with renal failure. Kidney Int. 2004;66(6):2237-44.

37. Maher ER, Young G, Smyth-Walsh B, Pugh S, Curtis JR. Aortic and mitral valve calcification in patients with end-stage renal disease. Lancet. 1987;2(8564):875-7.

38. Rubel JR, Milford EL. The relationship between serum calcium and phosphate levels and cardiac valvular procedures in the hemodialysis population. Am J Kidney Dis. 2003;41(2):411-21.

39. Schwarz U, Buzello M, Ritz E, Stein G, Raabe G, Wiest G, et al. Morphology of coronary atherosclerotic lesions in patients with end-stage renal failure. Nephrol Dial Transplant. 2000;15(2):218-23.

40. Levin A, Thompson CR, Ethier J, Carlisle EJ, Tobe S, Mendelssohn D, et al. Left ventricular mass index increase in early renal disease: impact of decline in hemoglobin. Am J Kidney Dis. 1999:34(1):125-34.

41. Silverberg DS, Wexler D, laina A. The importance of anemia and its correction in the management of severe congestive heart failure. Eur J Heart Fail. 2002;4(6):681-6.
42. Astor BC, Coresh J, Heiss G, Pettitt D, Sarnak MJ. Kidney function and anemia as risk factors for coronary heart disease and mortality: the atherosclerosis risk in communities (ARIC) study. Am Heart J. 2006;151(2): 492-500.

43. Levin A, Djurdjev O, Duncan J, Rosenbaum D, Werb R. Haemoglobin at time of referral prior to dialysis predicts survival: an association of haemoglobin with long-term outcomes. Nephrol Dial Transplant. 2006;21(2):370-7.

44. Ksiazek A, Zaluska WT, Ksiazek P. Effect of recombinant human erythropoietin on adrenergic activity in normotensive hemodialysis patients. Clin Nephrol. 2001;56(2):104-10.

45. Parfrey PS, Foley RN, Harnett JD, Kent GM, Murray DC, Barre PE. Outcome and risk factors for left ventricular disorders in chronic uraemia. Nephrol Dial Transplant. 1996;11(7):1277-85.

46. Damen JA, Hooft L, Schuit E, Debray TP, Collins GS, Tzoulaki I, et al. Prediction models for cardiovascular disease risk in the general population: systematic review. BMJ. 2016;353:i2416.

47. Danesh J, Collins R, Appleby P, Peto R. Association of fibrinogen, C-reactive protein, albumin, or leukocyte count with coronary heart disease: metaanalyses of prospective studies. JAMA. 1998;279(18):1477-82.

48. Zoccali C, Benedetto FA, Mallamaci F, Tripepi G, Giacone G, Cataliotti A, et al. Prognostic value of echocardiographic indicators of left ventricular systolic function in asymptomatic dialysis patients. J Am Soc Nephrol. 2004; 15(4):1029-37.

49. deFilippi C, Wasserman S, Rosanio S, Tiblier E, Sperger H, Tocchi M, et al. Cardiac troponin $\mathrm{T}$ and $\mathrm{C}$-reactive protein for predicting prognosis, coronary atherosclerosis, and cardiomyopathy in patients undergoing long-term hemodialysis. JAMA. 2003;290(3):353-9.

\section{Publisher's Note}

Springer Nature remains neutral with regard to jurisdictional claims in published maps and institutional affiliations.

\section{Ready to submit your research? Choose BMC and benefit from:}

- fast, convenient online submission

- thorough peer review by experienced researchers in your field

- rapid publication on acceptance

- support for research data, including large and complex data types

- gold Open Access which fosters wider collaboration and increased citations

- maximum visibility for your research: over $100 \mathrm{M}$ website views per year

At BMC, research is always in progress.

Learn more biomedcentral.com/submissions 